\title{
Modular Planar Antenna at X-band for satellite communications
}

\section{J.M. Inclán-Alonso ${ }^{1}$, A. García-Aguilar ${ }^{1}$, L. Vigil-Herrero ${ }^{1}$, J.M. Fernández-González ${ }^{1}$, J. Sanmartín-Jara ${ }^{2}$ and $^{-}$ M. Sierra-Pérez ${ }^{1}$}

\author{
${ }^{1}$ Radiation Group. Signals, Systems and Radiocommunications Dept. \\ Universidad Politécnica de Madrid. Ciudad Universitaria. 28040. Madrid, Spain. \\ \{ chema, andresg, jmfdez, m.sierra.perez $\}$ gr.ssr.upm.es \\ ${ }^{2}$ Antenas Moyano CO. LTD.
}

\begin{abstract}
An antenna which has been conceived as a portable system for satellite communications based on the recommendations ITU-R S.580-6 [1] and ITU-R S.465-5 [2] for small antennas, i.e., with a diameter lower than 50 wavelengths, is introduced. It is a planar and a compact structure with a size of $40 \times 40 \times 2 \mathrm{~cm}$. The antenna is formed by an array of 256 printed elements covering a large bandwidth (14.7\%) at X-Band. The specification includes transmission $(\mathrm{Tx})$ and reception $(\mathrm{Rx})$ bands simultaneously. The printed antenna has a radiation pattern with a $3 \mathrm{~dB}$ beamwidth of $5^{\circ}$, over a $31 \mathrm{dBi}$ gain, and a dual and an interchangeable circular polarization.
\end{abstract}

\section{Introduction}

The antenna is formed by array of 16x16 printed elements and covers a large band. The antenna has been divided into $4 \times 4$ subarrays, therefore each subarray has $4 \times 4$ radiating elements. Subarrays are composed of several layers. The bottom layer is a microstrip network that contains the distribution network for RHCP and LHCP and hybrid circuits to get circular polarization. The upper layers of the subarray contain the double stacked patches.

To distribute the power among the subarrays a low losses stripline distribution network is used. In order to get a low side lobes level, the subarrays of the corners receive less power that the ones in the center as it can be shown in Fig 1(a).

The subarrays are connected to the stripline through SMP-type coaxial connectors. In the power distribution network there are two inputs/outputs in SMA-type connector, one for each polarization. The structure can be shown in Fig 1(b).

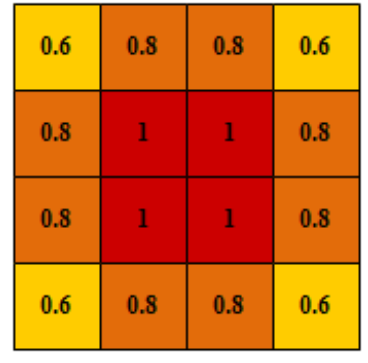

(a)

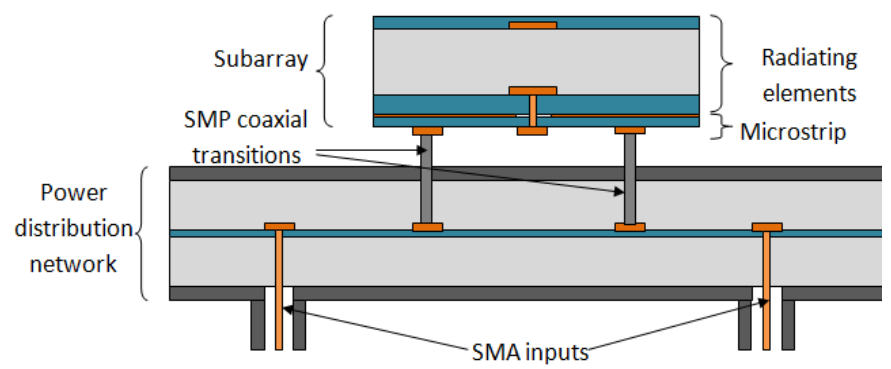

(b)

Fig 1.Subarray distribution feedings (a) and Antenna structure (b)

\section{Radiating element}

The radiating element is a double stacked circular patch. In order to get a circular polarization the patch is excited by two coaxial probes with orthogonal signals. To provide the antenna with dual polarization in transmission and in reception a hybrid circuit is used. This hybrid circuit is placed in the microstrip layer as is shown in Fig 2.

Patches are printed in a PTFE_Glass substrate with a permittivity of 2.17. The actives patches are separated from the parasites patches by a thick sheet of foam.

The comparison between the simulated S-parameters and the measured S-parameters at the lower band can be shown Fig 3. It is important to stress out that due to the behaviour of the hybrid circuit the measured S11 represents the coupling between coaxial probes of each polarization and S21 represents mainly the adaptation of the patch.

The coupling between polarizations is high in the measures and in the simulation. This is caused by the height of the substrate of the active patches and the coupling between the coaxial probes. This coupling between polarizations can 
lead to high side lobes level and worse CP/XP ratio. To reduce the influence of this coupling Willkinson power dividers are used in microstrip network.

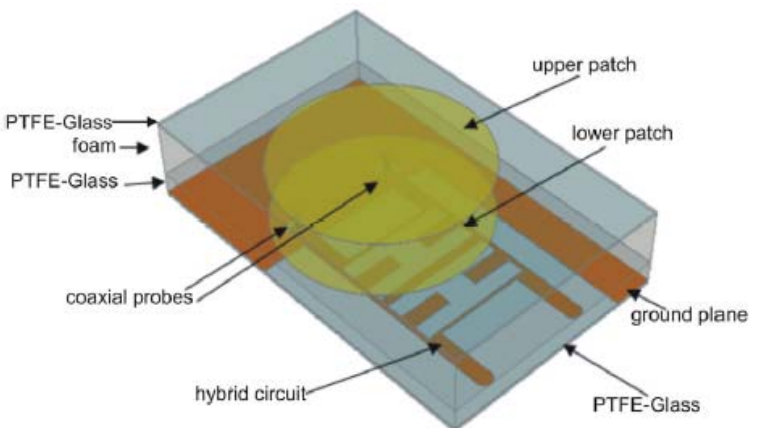

(a)
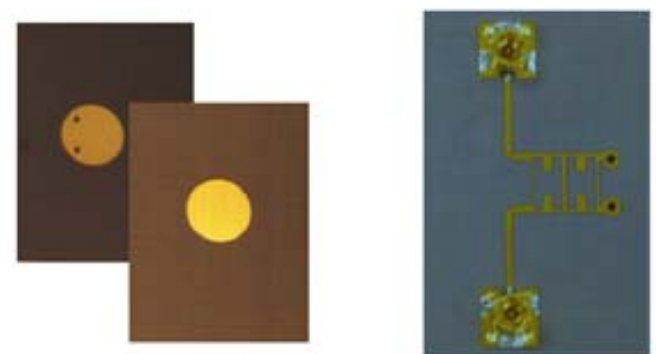

(b)

Fig 2. Radiating element structure (a) and test-board of radiating element

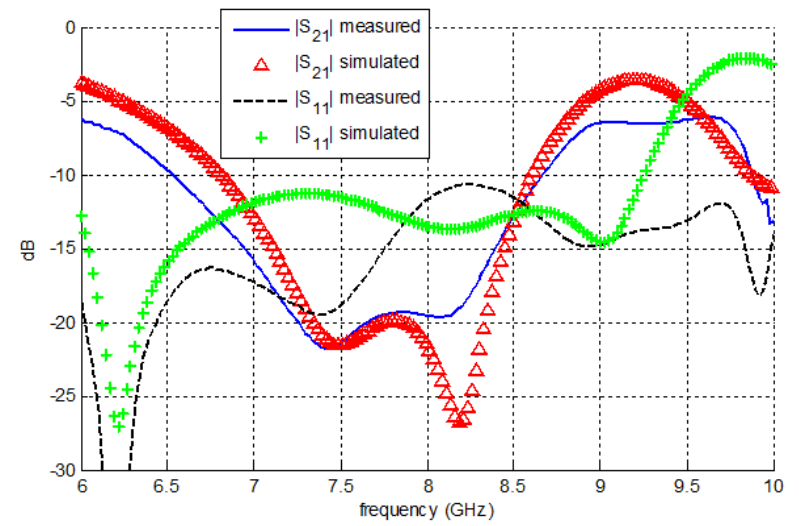

(a)

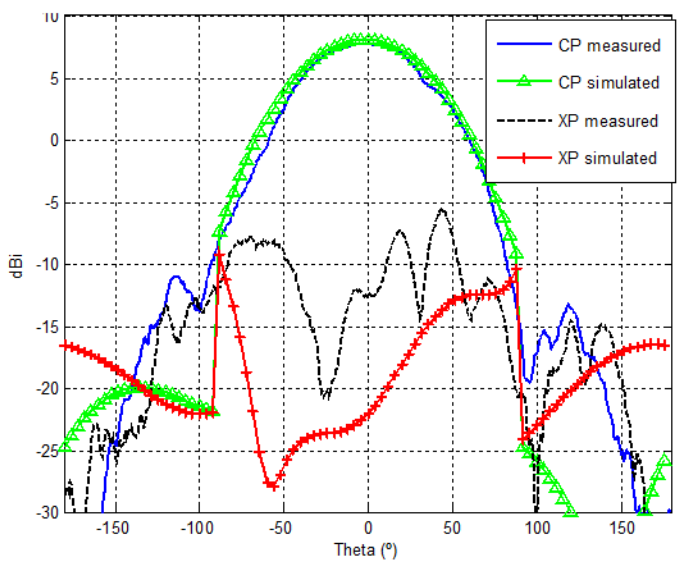

(b)

Fig 3. S-Parameter (a) and radiation pattern (b) of the radiating element

\section{Subarray system}

Each subarray has $4 \times 4$ radiating elements separated by $25 \mathrm{~mm}$. The distribution network among the radiating elements is done in microstrip technology. RHCP and LHCP network are implemented in the same layer. The size of the subarrays in the first prototypes is 99x99 mm. To improve the purity of the polarization the sequential rotation technique is implemented among each 4 elements as it is shown in Fig 4. Thus, each element is rotated $90^{\circ}$ and excited by a $90^{\circ}$ phase-shifted signal compared to the previous one.
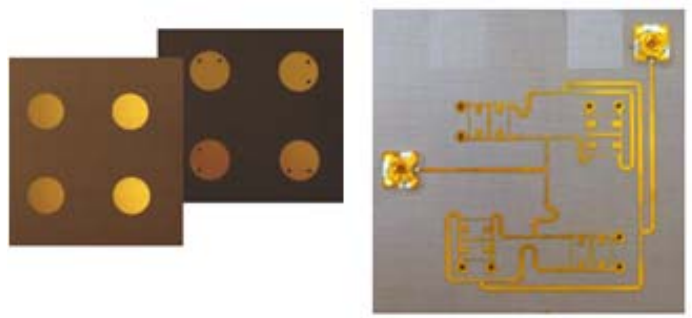

Fig 4. Test-board of the array unit cell

The results of a unit cell (2x2 elements) are shown in Fig 5. As we can see the axial ratio is bellow $1.3 \mathrm{~dB}$ in $\mathrm{X}$ band. 


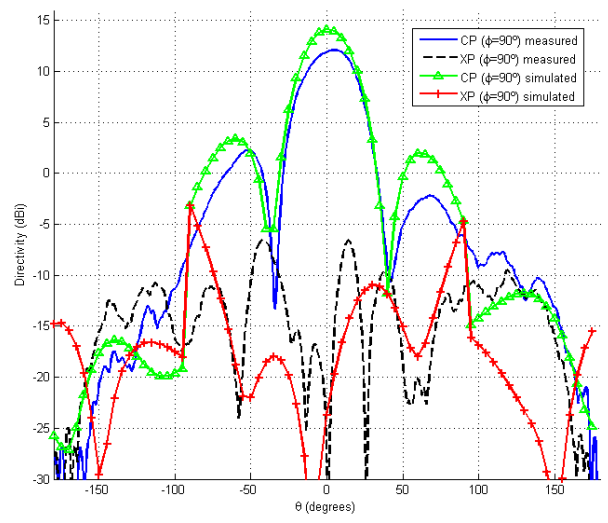

(a)

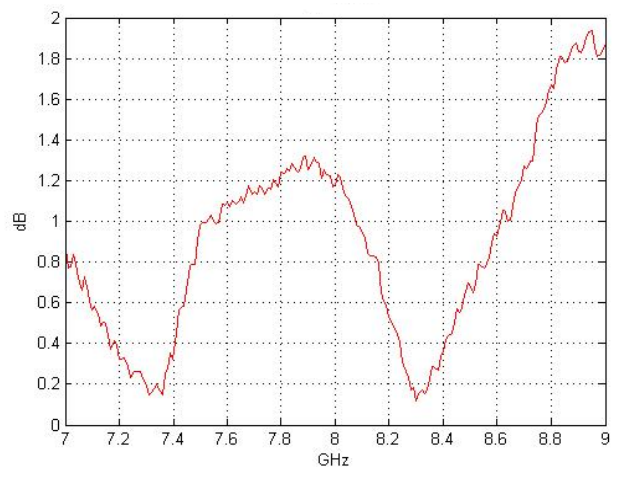

(b)

Fig 5. Radiation pattern of the unit cell at $7.25 \mathrm{GHz}$ (a) and axial relation of the unit cell (b)

\subsection{Power distribution network}

A stripline has been used to distribute the power among the subarrays in order to get a low loss network. The stripline is formed by a metalized line in a thin substrate located between two foam sheets and two plates, which are the ground plane. A quasi-TEM mode is propagated due to the presence of two different dielectrics (PTFE and foam). In order to have a low loss distribution network the height of the foam sheets must be high.

The transitions between stripline and each subarray are done through SMP type coaxial connectors. The two inputs of the antenna, one for each polarization, are done through SMA type coaxial connectors. To avoid the generation of a TE mode in vertical transitions it is necessary to place a short-ended pseudo-waveguide thereby the TE mode stays as a cut-off mode. The losses for each transition are around $0.3 \mathrm{~dB}$. The S-parameters of the transition between the stripline and SMP-Connectors are shown in Fig 6. In the S21 parameter the losses in the two SMP connectors are included.
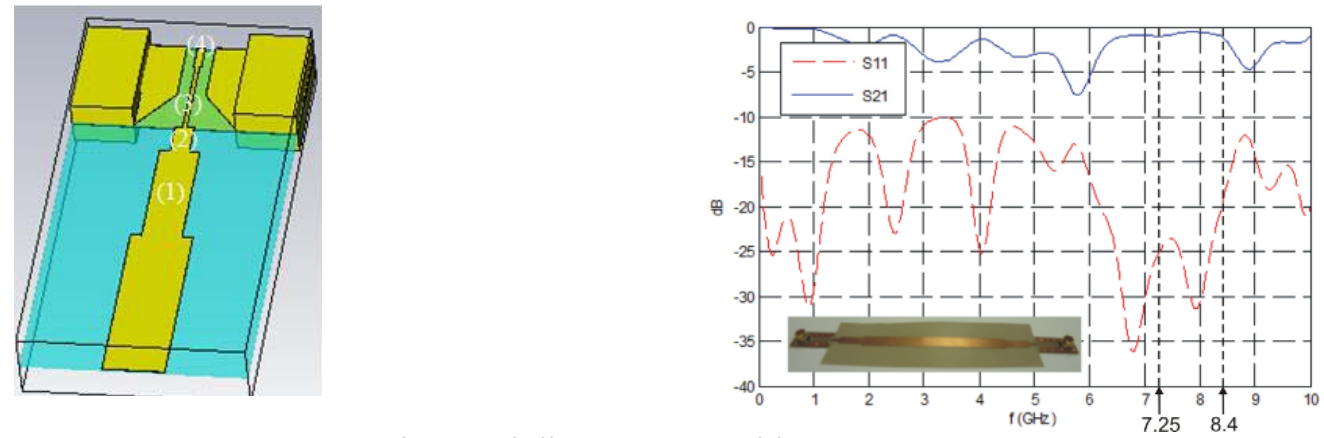

Fig 6. Stripline-.SMP transition

The first prototype of the power distribution network is shown in Fig 7. We have detected some unexpected problems in the first prototype. The main problem is that a TE resonant mode is excited not only in the vertical transitions but also in other parts of the line. This makes that peaks in the transmission to each subarray appear in some frequencies. To solve this problem a protected stripline network is chosen (second prototype of Fig 7).
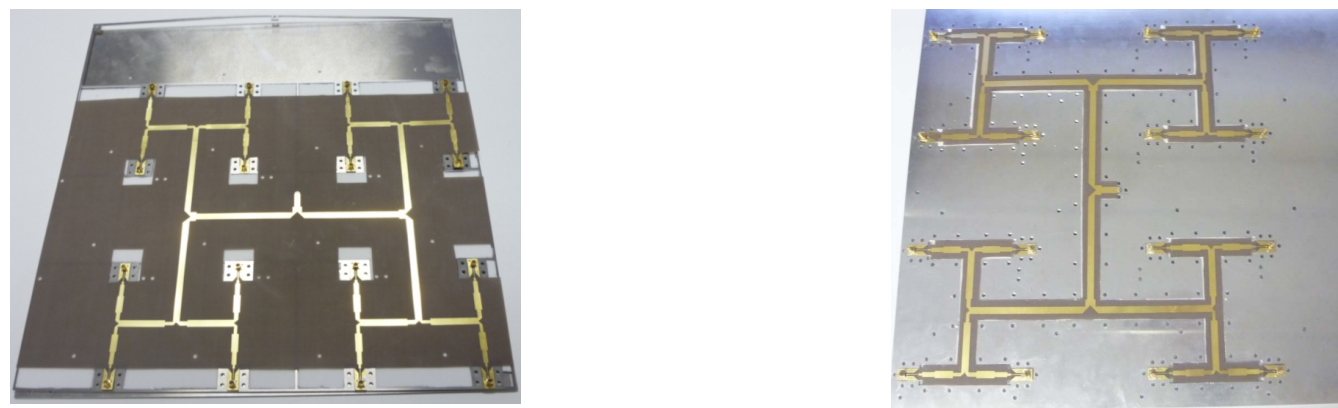

Fig 7. Fist and second prototype of the power distribution network 


\section{Results}

The radiation pattern for the first prototype is shown in Fig 8 . The total losses in the whole antenna are about $4 \mathrm{~dB}$ at the lower band. In the first prototype we have also detected that there are couplings between the polarizations due to the small distance between some lines in the subarray network. For this reason in the second prototype a $3 \times 3$ subarray antenna will be implemented, making bigger the size of each subarray.

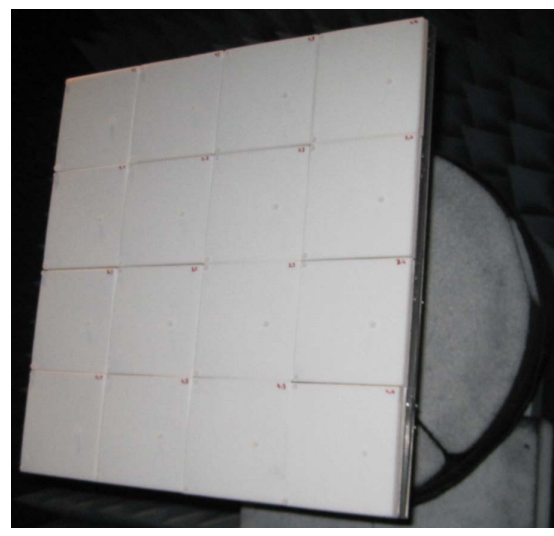

(a)

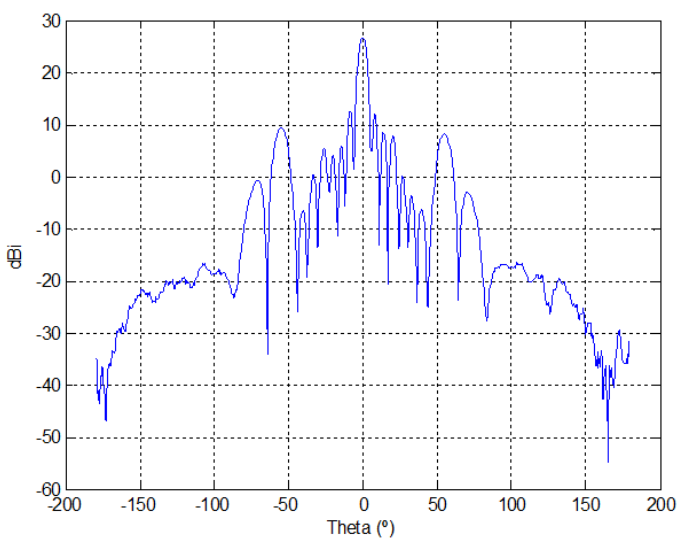

(b)

Fig 8. First prototype (a) and radiation pattern at $7.4 \mathrm{GHz}(\mathrm{b})$

\section{Conclusion}

The complete design of a portable printed antenna for satellite communications at X-Band has been introduced. This antenna has been conceived as a planar, compact, modular, low losses and dual circular polarized antenna for Tx and Rx bands simultaneously. First prototype was designed and built successfully. In order to solve some problems detected in the first prototype a second prototype has been designed.

\section{Acknowledgments}

The simulations done in this work has been realized using CST Microwave Studio Suite 2010 under a cooperation agreement between Computer Simulation Technology (CST) and Universidad Politécnica de Madrid. The project is leadered by the Universidad Politécnica de Madrid and Antenas Moyano SL and the support of the Spanish Education Ministry (Comisión Interministerial de Ciencia y Tecnología under reference TEC2008-06736/TEC, a Spanish government scholarship (FPI under reference BES-2009-021462), a Universidad Politécnica de Madrid scholarship and the help of European Cost Action IC-0603 Assist. The substrate used in the prototypes was donated by NELTEC S.A.

\section{References}

1. ITU-R Recommendation S.580-6 (2004). Radiation diagrams for use as design objectives for antennas of earth stations operating with geostationary satellites.

2. ITU-R Recommendation S.465-5 (1993), Reference earth-station radiation pattern for use in coordination and interference assessment in the frequency range from 2 to about $30 \mathrm{GHz}$.

3. M. Sierra-Castañer, M. Vera-Isasa, M. Sierra-Pérez, J.L. Fernández-Jambrina (2005), Double-Beam Parallel Plate Slot Antenna, IEEE Transactions on Antennas and Propagation, vol. 53, no. 3, pp. 977-984.

4. Matthaei, L. Young, E.M.T. Jones, "Microwave filters, impedance-matching networks, and coupling structures", Artech House, 1980. 